\title{
Anomalous fluctuations of the condensate in interacting Bose gases
}

\author{
S. Giorgini ${ }^{1,4}$, L. P. Pitaevskii ${ }^{2,3,4}$ and S. Stringari ${ }^{4}$ \\ ${ }^{1}$ European Centre for Theoretical Studies in Nuclear Physics and Related Areas \\ Villa Tambosi, Strada delle Tabarelle 286, I-38050 Villazzano, Italy \\ ${ }^{2}$ Department of Physics, Technion, 32000 Haifa, Israel \\ ${ }^{3}$ Kapitza Institute for Physical Problems, 117454 Moscow, Russia \\ ${ }^{4}$ Dipartimento di Fisica, Università di Trento, \\ and Istituto Nazionale di Fisica della Materia, I-38050 Povo, Italy
}

We find that the fluctuations of the condensate in a weakly interacting Bose gas confined in a box of volume $V$ follow the law $\left\langle\delta N_{0}^{2}\right\rangle \sim V^{4 / 3}$. This anomalous behaviour arises from the occurrence of infrared divergencies due to phonon excitations and holds also for strongly correlated Bose superfluids. The analysis is extended to an interacting Bose gas confined in a harmonic trap where the fluctuations are found to exhibit a similar anomaly.

02.70.Lq, 67.40.Db

The recent experimental achievement [1] of BoseEinstein condensation (BEC) in dilute atomic vapours has stimulated a great revival of interest in the theoretical study of this phenomenon (for un update list of references see [2]). Among the several intriguing questions associated with Bose-Einstein condensation the problem of fluctuations plays an important role in the more general context of statistical mechanics (see for example Ref. [3]).

The fluctuations of the condensate have been studied in a systematic way in the case of the ideal Bose gas in a box [4,5], and, more recently, in the presence of a harmonic trap [6 8]. In the ideal Bose gas one finds a dramatically different behaviour between the canonical and the grand canonical ensembles. In particular the results of Ref. [. 3 . show that in the canonical ensemble the fluctuations of the number of particles in the ground state of a box of volume $V$ behave as $V^{4 / 3}$. This law ensures that the relative fluctuations $\left\langle\left(\delta N_{0}\right)^{2}\right\rangle / V^{2}$ of the condensate vanish when $V \rightarrow \infty$, differently from what happens in the grand canonical ensemble, where $\left\langle\left(\delta N_{0}\right)^{2}\right\rangle=N_{0}^{2}+N_{0} \sim V^{2}$. The results obtained in the canonical ensemble, however, reveal that the fluctuations of the condensate are not linear in $V$, in contrast to the fluctuations of most physical observables. One might think that this anomalous behaviour is a peculiarity of the ideal Bose gas and that, as soon as one includes interaction effects, the fluctuations of $N_{0}$ take a normal behaviour, linear in $V$. In this letter we address explicitly this question and we find that, despite the finite compressibility of the system, the fluctuations of the condensate still behave as $V^{4 / 3}$.

Let us start our investigation in the framework of Bo- goliubov theory of a uniform weakly interacting Bose gas confined in a cubic box of size $L$. According to Bogoliubov theory [9] the particle operator can be expressed in terms of the quasi-particle creation and annihilation operators as $a_{\mathbf{p}}=u_{p} \alpha_{\mathbf{p}}+v_{p} \alpha_{-\mathbf{p}}^{\dagger}$, where $\mathbf{p} \neq 0$ is the eigenvalue of the momentum operator $\mathbf{p}=2 \pi \hbar \mathbf{n} / L$, with $\mathbf{n}=\left(n_{x}, n_{y}, n_{z}\right)$. The real quantities $u_{p}$ and $v_{p}$ satisfy the relations

$$
\begin{aligned}
& u_{p}^{2}+v_{p}^{2}=\frac{\left(\epsilon_{p}^{2}+\mathrm{g}^{2} n_{0}^{2}\right)^{1 / 2}}{2 \epsilon_{p}} \\
& u_{p} v_{p}=-\frac{\mathrm{g} n_{0}}{2 \epsilon_{p}}
\end{aligned}
$$

and, consequently, the normalization condition $u_{p}^{2}-v_{p}^{2}=$ 1. The energy of the elementary excitations entering (1) are given by the most famous Bogoliubov spectrum

$$
\epsilon_{p}=\left(\left(\frac{p^{2}}{2 m}+\mathrm{g} n_{0}\right)^{2}-\mathrm{g}^{2} n_{0}^{2}\right)^{1 / 2}
$$

where $\mathrm{g}=4 \pi \hbar^{2} a / m$ is the coupling constant fixed by the s-wave scattering length $a$, and $n_{0}$ is the condensate density which, at low $T$, coincides with the total density $n=N / V$, apart from higher order effects in $\mathrm{g}$ arising from the quantum depletion of the condensate. At small $p$ eq. (2) gives the phonon dispersion law $\epsilon_{p}=c p$ with the sound velocity given by $c=\sqrt{\mathrm{g} n_{0} / m}$. Notice that, due to the discretization of the values of $p$, the phonon dispersion law is ensured only if the condition $N a / L \gg 1$ is satisfied.

The total number of particles out of the condensate is given by the expectation value of the operator

$$
\begin{aligned}
N_{\text {out }}=\sum_{\mathbf{p} \neq 0} a_{\mathbf{p}}^{\dagger} a_{\mathbf{p}} & =\sum_{\mathbf{p} \neq 0}\left[u_{p}^{2} \alpha_{\mathbf{p}}^{\dagger} \alpha_{\mathbf{p}}+v_{p}^{2} \alpha_{\mathbf{p}} \alpha_{\mathbf{p}}^{\dagger}\right. \\
& \left.+u_{p} v_{p}\left(\alpha_{\mathbf{p}} \alpha_{-\mathbf{p}}+\alpha_{\mathbf{p}}^{\dagger} \alpha_{-\mathbf{p}}^{\dagger}\right)\right]
\end{aligned}
$$

and takes the form

$$
\left\langle N_{\text {out }}\right\rangle=\sum_{\mathbf{p} \neq 0}\left(\left(u_{p}^{2}+v_{p}^{2}\right) f_{p}+v_{p}^{2}\right),
$$

where

$$
f_{p}=\left\langle\alpha_{\mathbf{p}}^{\dagger} \alpha_{\mathbf{p}}\right\rangle=\left(\exp \epsilon_{p} / k_{B} T-1\right)^{-1}
$$


is the number of elementary excitations present in the system at thermal equilibrium. Notice that the number of thermally excited atoms differs from the number of elementary excitations because of the factor $\left(u_{p}^{2}+v_{p}^{2}\right)$. In the thermodynamic limit $(V \rightarrow \infty)$ the sum (4) can be calculated by integration in momentum space. At low temperature this yields the result

$$
\left\langle N_{\text {out }}\right\rangle=N\left(\frac{m\left(k_{B} T\right)^{2}}{12 n c \hbar^{3}}+\frac{8 \sqrt{\pi}}{3} \sqrt{a^{3} n}\right),
$$

where $N$ is the total number of particles. The term proportional to $T^{2}$ accounts for the phonon contribution to thermal fluctuations [10], while the temperature independent term gives the effect of quantum fluctuations [9]. The depletion of the condensate is obtained by simply taking the difference $N_{0}=N-\left\langle N_{\text {out }}\right\rangle$. For the ideal Bose gas confined in a box $\left[u_{p}=1, v_{p}=0\right.$ in (4) $]$ the same procedure gives the well known result $\left\langle N_{\text {out }}\right\rangle=N\left(T / T_{c}\right)^{3 / 2}$, where $k_{B} T_{c}=2 \pi \hbar^{2} / m(n / \zeta(3 / 2))^{2 / 3}$ is the Bose-Einstein transition temperature.

In a similar way one can proceed to the calculation of the fluctuations of $N_{\text {out }}$. Starting from (3) one finds

$$
\begin{aligned}
\left\langle\delta N_{\text {out }}^{2}\right\rangle & =\sum_{\mathbf{p} \neq 0}\left[\left(\left(u_{p}^{2}+v_{p}^{2}\right)^{2}+4 u_{p}^{2} v_{p}^{2}\right)\left(f_{p}^{2}+f_{p}\right)\right] \\
& +2 \sum_{\mathbf{p} \neq 0} u_{p}^{2} v_{p}^{2}
\end{aligned}
$$

To calculate the expectation value of the terms with four quasi-particle operators appearing in $\left\langle N_{\text {out }}^{2}\right\rangle$ we have used Wick's theorem

$$
\left\langle\alpha_{\mathbf{p}}^{\dagger} \alpha_{\mathbf{p}} \alpha_{\mathbf{p}^{\prime}}^{\dagger} \alpha_{\mathbf{p}^{\prime}}\right\rangle=\left\langle\alpha_{\mathbf{p}}^{\dagger} \alpha_{\mathbf{p}}\right\rangle\left\langle\alpha_{\mathbf{p}^{\prime}}^{\dagger} \alpha_{\mathbf{p}^{\prime}}\right\rangle+\left\langle\alpha_{\mathbf{p}}^{\dagger} \alpha_{\mathbf{p}^{\prime}}\right\rangle\left\langle\alpha_{\mathbf{p}} \alpha_{\mathbf{p}^{\prime}}^{\dagger}\right\rangle,
$$

which holds for the creation and annihilation operators of statistically independent excitations. In the ideal Bose gas $\left(u_{p}=1, v_{p}=0\right)$ result (7) is correct at all temperatures if one works in the grand canonical ensemble, and, as demonstrated in Ref. [3], holds also in the canonical ensemble except near and, of course, above $T_{c}$. Physically this behaviour follows from the fact that the condensate plays the role of a "reservoir" for the particles out of the condensate. On the other hand, in interacting Bose systems eq. (7) is valid only at low temperature where the number of excitations is small and their interaction can be neglected. Both in the interacting and non-interacting case eq. (7) allows us to calculate the fluctuations of the condensate in the canonical ensemble where one has trivially $\left\langle\delta N_{0}^{2}\right\rangle=\left\langle\delta N_{\text {out }}^{2}\right\rangle$ 11.

In equation $(7)$ one immediately points out the occurrence of a strong infrared divergency arising from the term proportional to $f_{p}^{2}$. In fact at low $p$ one has $u_{p}^{2} \simeq v_{p}^{2} \simeq \mathrm{g} n_{0} / 2 c p$ and $f_{p} \simeq k_{B} T / c p$. This results in a $1 / p^{4}$ infrared divergency yielding a divergent integral in $3 \mathrm{D}$. Also in the ideal gas one finds a similar behaviour. In fact in this case $u_{p}^{2}=1, v_{p}^{2}=0$, while $f_{p} \simeq 2 m k_{B} T / p^{2}$.
The occurrence of this divergency implies that the sum (7) cannot be calculated by integration in momentum space, but is dominated by the discretized sum over the low-energy phonon modes. The final result for the leading terms in the large- $N$ limit is given by

$$
\begin{aligned}
\left\langle\delta N_{0}^{2}\right\rangle & =A\left(\frac{m k_{B} T}{\hbar^{2}}\right)^{2} V^{4 / 3} \\
& +\frac{1}{3 \pi^{2}} \frac{k_{B} T m^{2} c}{\hbar^{3}} V \log \left(\frac{V m^{3} c^{3}}{\hbar^{3}}\right)+o(V)
\end{aligned}
$$

where $A=2 /(\pi)^{4} \sum_{\mathbf{n} \neq 0} 1 / \mathbf{n}^{4}=0.105$. The term containing $\log V$ arises from the contribution linear in $f_{p}$ of eq. (7) which yields an integral in momentum space exhibiting a logarithmic divergency. It is worth noting that the coefficient of the leading term does not depend on the velocity of sound (and hence on the interaction coupling constant), nor on the number of particles. In the ideal Bose gas (IBG) using cyclic boundary conditions the result looks similar, but the coefficient differs by a factor 2 12

$$
\left\langle\delta N_{0}^{2}\right\rangle_{C}^{I B G}=2 A\left(\frac{m k_{B} T}{\hbar^{2}}\right)^{2} V^{4 / 3}+o(V) .
$$

In this case it is important to specify that the result holds in the canonical (C) ensemble. The IBG result (9) holds if the condition $\mathrm{Na} / L \ll 1$ is satisfied.

The anomalous behaviour (8) exhibited by the fluctuations of the condensate reflects the occurrence of infrared divergencies in the one-particle Green's function [13. These phenomena are well accounted for by the hydrodynamic theory of Bose superfluids (see for example Ref. 14). Actually this theory can be directly employed to derive result (8) which consequently holds not only in the case of a weakly-interacting Bose gas, but also in strongly-interacting Bose fluids, like liquid ${ }^{4} \mathrm{He}$. Notice that in this case the condensate fraction at $T=0$ sizably differs from the ideal gas value and that the sound velocity $c$, entering the $\log V$ term in eq. (8), cannot be calculated using Bogoliubov theory.

The fluctuations of the condensate can also be evaluated at $T=0\left[f_{p}=0\right.$ in eq. (17)]. In this case the sum has no infrared divergencies and can be safely calculated through direct integration in momentum space. This yields the result

$$
\left\langle\delta N_{0}^{2}\right\rangle(T=0)=\sqrt{\pi}\left(a n_{0}\right)^{3 / 2} V,
$$

revealing that, at $T=0$, the fluctuations of the condensate have a "normal" behaviour, i.e. they are linear in $V$. These fluctuations are however large compared to the ones of the total number of particles, which in the grand canonical ensemble behave as $\left\langle\delta N^{2}\right\rangle \simeq V^{2 / 3} \log V$. This latter result is obtained by writing

$$
\left\langle\delta N^{2}\right\rangle=n \int d \mathbf{r}_{1} d \mathbf{r}_{2} \nu\left(\left|\mathbf{r}_{2}-\mathbf{r}_{1}\right|\right)
$$


where $\nu(r)$ is the density-density static correlation function $n \nu\left(\left|\mathbf{r}_{2}-\mathbf{r}_{1}\right|\right) \equiv\left\langle\delta n\left(\mathbf{r}_{2}\right) \delta n\left(\mathbf{r}_{1}\right)\right\rangle$. In an interacting Bose superfluid at $T=0$ the large $r$ behaviour of $\nu(r)$ is fixed by the asymptotic law 15]

$$
\lim _{r \rightarrow \infty} \nu(r)=-\frac{\hbar}{2 \pi^{2} m c} \frac{1}{r^{4}} .
$$

Let us calculate the fluctuations of $N$ in a sphere of radius $R \gg \hbar / m c$. The domain of integration in (11) is then $r_{1}, r_{2} \leq R$ and one can write

$$
\left\langle\delta N^{2}\right\rangle=4 \pi n \int_{0}^{2 R} d r r^{2} \tau(r) \nu(r)
$$

where $\tau(r)=2 \pi R^{3}\left(2-3 r / 2 R+r^{3} / 8 R^{3}\right) / 3$ is the overlapping volume of two spheres of radius $R$ whose centers are separeted by the distance $r$. The integral of the $r$ independent term in $\tau(r)$ gives a contribution proportional to $R^{2}$ as can be seen by exploiting the sum rule $4 \pi \int_{0}^{\infty} d r r^{2} \nu(r)=S(k=0)=0$, holding for systems where the static form factor $S(k)$ vanishes in the longwavelength limit. The dominant contribution to $\left\langle\delta N^{2}\right\rangle$ comes thus from the term linear in $r$ in $\tau(r)$. One finally finds the relevant result 16 ]

$$
\left\langle\delta N^{2}\right\rangle(T=0)=\frac{2 n \hbar}{m c} R^{2} \log \left(\frac{R m c}{\hbar}\right),
$$

holding with logarithmic accuracy.

The analysis developed above can be easily extended to the case of a Bose gas confined in a harmonic potential of the form $V_{\text {ext }}=m \omega_{0}^{2} r^{2} / 2$, where, for simplicity, we have considered isotropic trapping. Also in this case one can define a proper "thermodynamic limit". This is achieved by letting $\omega_{0} \rightarrow 0, N \rightarrow \infty$ while keeping $\omega_{0}^{3} N$ fixed 18, 19. Differently from the gas confined in a box, in the presence of harmonic trapping the density of the system is inhomogeneous and it is convenient to use the following decomposition for the particle field operator

$$
\psi(\mathbf{r})=\Phi(\mathbf{r})+\sum_{i}\left(u_{i}(\mathbf{r}) \alpha_{i}+v_{i}^{*}(\mathbf{r}) \alpha_{i}^{\dagger}\right),
$$

where $\Phi(\mathbf{r})=\langle\psi(\mathbf{r})\rangle$ is the order parameter and the index $i$ labels the elementary excitations of the system for which the normalization condition $\int d \mathbf{r}\left(\left|u_{i}\right|^{2}-\left|v_{i}\right|^{2}\right)=1$ holds. The number of particles out of the condensate is given by the operator $N_{\text {out }}=\int d \mathbf{r}\left(\psi^{\dagger}(\mathbf{r})-\Phi^{*}(\mathbf{r})\right)(\psi(\mathbf{r})-\Phi(\mathbf{r}))$.

Let us first discuss the non-interacting gas. In this case the fluctuations of $N_{\text {out }}$ can be written as $\left\langle\delta N_{\text {out }}^{2}\right\rangle=$ $\sum_{i} f_{i}\left(f_{i}+1\right)$, where $i$ labels the single-particle excited states of the oscillator Hamiltonian. By using the semiclassical approximation for these states [18], we obtain the result [6]

$$
\left\langle\delta N_{0}^{2}\right\rangle_{C}^{I B G}=\frac{\pi^{2}}{6 \zeta(3)} N\left(\frac{T}{T_{c}}\right)^{3}
$$

for the fluctuations of the condensate in the canonical ensemble. Result (16) holds for temperatures smaller than the critical temperature for BEC [17]

$$
k_{B} T_{c}=\hbar \omega_{0}\left(\frac{N}{\zeta(3)}\right)^{1 / 3}
$$

which is kept fixed in the thermodynamic limit. The applicability of the semiclassical approximation is ensured for temperatures much larger than the oscillator temperature $\left(k_{B} T \gg \hbar \omega_{0}\right)$ and is a consequence of the fact that the sum $\sum_{i} f_{i}\left(f_{i}+1\right)$ takes most of its contribution from excitations with energy $\sim k_{B} T$. The changes in the fluctuations of the condensate (16) due to the use of the microcanonical ensemble can be also calculated and result in a change of the numerical prefactor [7,8].

Result (16) shows that the fluctuations of the condensate in a harmonic trap have a normal behaviour, i.e. they are proportional to $N$. This behaviour differs from result (9) for the ideal Bose gas in a box where the fluctuations have a stronger dependence on $N$ (or $V$ ). This fact is not a surprise because it is well known that confinement reduces the effects of thermal fluctuations. For example, the 2D ideal Bose gas in a harmonic trap exhibits Bose-Einstein condensation at finite temperature, differently from what happens in the uniform gas where thermal fluctuations have a destabilizing effect on the condensate.

Interactions introduce however a deep difference in the above behaviour. In fact for repulsive forces the system exhibits a phonon-type behaviour at low excitation energies. This suggests that the fluctuations of the condensate will be dominated by the discretized low energy modes which in the large- $N$ limit obey the dispersion law 20

$$
\epsilon(n, \ell)=\hbar \omega_{0}\left(2 n^{2}+2 n \ell+3 n+\ell\right)^{1 / 2} .
$$

These phonon-like collective excitations have been recently measured [21] and the observed frequencies are in excellent agreement with theory. Let us recall that the dispersion law (18) is valid if the conditions $N a / a_{H O} \gg 1$ and $\epsilon(n, \ell) \ll \mu$ are satisfied. Here $a_{H O}=\sqrt{\hbar / m \omega_{0}}$ is the oscillator length and $\mu$ is the chemical potential calculated at zero temperature. The condition $N a / a_{H O} \gg 1$, which is well satisfied in the recent experiments on BEC, is the analogue of the condition $N a / L \gg 1$ which must be satisfied in the transition from the ideal to the Bogoliubov gas in the box. Furthermore, the above condition yields the "Thomas-Fermi" result for the ground state density of the system $n_{0}(\mathbf{r})=|\Phi(\mathbf{r})|^{2}=\mu / \mathrm{g}(1-$ $\left.r^{2} / R_{c}^{2}\right) \theta\left(R_{c}-r\right)$, where $R_{c}=2 \mu /\left(m \omega_{0}^{2}\right)$ is the radius of the condensate and $\mu=\hbar \omega_{0}\left(15 N a / a_{H O}\right)^{2 / 5} / 2$. In the same limit one can also calculate the quantities $u_{i}$ and $v_{i}$ of (15) relative to the low-lying excitations (18). One obtains the following leading behaviour [22] 


$$
u_{i}(\mathbf{r}) \simeq-v_{i}(\mathbf{r}) \simeq \sqrt{\frac{\mathrm{g} n_{0}(\mathbf{r})}{2 \epsilon_{i}}} \chi_{i}(\mathbf{r}),
$$

where $\chi_{i}(\mathbf{r})$ is the velocity potential associated with the collective mode satisfying the condition $\int d \mathbf{r} \chi_{i}^{*}(\mathbf{r}) \chi_{j}(\mathbf{r})=$ $\delta_{i j}$. After a straightforward calculation one finds that the leading term in the fluctuations of the condensate exhibits an anomalous behaviour proportional to $N^{4 / 3}$ :

$$
\left\langle\delta N_{0}^{2}\right\rangle=B \frac{T^{2}}{T_{c}^{2}}\left(\frac{m a^{2} k_{B} T_{c}}{\hbar^{2}}\right)^{2 / 5} N^{4 / 3},
$$

where $T_{c}$ is given in (17) and $B$ is a dimensionless coefficient

$$
\begin{aligned}
B & =\gamma \sum_{n n^{\prime} \ell} \frac{(2 \ell+1)}{\left(2 n^{2}+2 n \ell+3 n+\ell\right)\left(2 n^{\prime 2}+2 n^{\prime} \ell+3 n^{\prime}+\ell\right)} \\
& \times \frac{\left(\int_{0}^{1} d x x^{2(\ell+1)}\left(1-x^{2}\right) P_{\ell}^{(2 n)} P_{\ell}^{\left(2 n^{\prime}\right)}\right)^{2}}{\int_{0}^{1} d x x^{2(\ell+1)}\left(P_{\ell}^{(2 n)}\right)^{2} \int_{0}^{1} d x x^{2(\ell+1)}\left(P_{\ell}^{\left(2 n^{\prime}\right)}\right)^{2}},
\end{aligned}
$$

with $\gamma=15^{4 / 5} /\left(2(\zeta(3))^{8 / 15}\right)$. In the above equation $n, n^{\prime}, \ell=0,1,2, .$. and $\ell \neq 0$ if $n\left(n^{\prime}\right)=0$, while $P_{\ell}^{(2 n)} \equiv P_{\ell}^{(2 n)}(x)$ are the polinomials introduced in Ref. [20]. It is worth noting that the inclusion of interaction reproduces a behaviour similar to the one exhibited by the gas confined in a box, i.e. proportional to $N^{4 / 3}$. This result reveals that the apparently similar behaviour in the ideal and interacting Bose gases in a box is accidental and that the physical effects induced by interactions show up in a clearer way in the presence of the harmonic trap.

The consequences of interactions on the fluctuations of the condensate are expected to be even more dramatic in the case of attractive forces, where for values of $N$ close to the critical size for the onset of instability, the monopole vibration has a vanishing frequency with consequent major contribution to the sum (20). This situation, which is characterized by a full instability of the grand canonical ensemble, will be considered in a subsequent work.

It is finally important to keep in mind that only the fluctuations of the condensate exhibit the anomalous behaviour discussed in the present work. In particular the fluctuations of the density operator $\psi^{\dagger}(\mathbf{r}) \psi(\mathbf{r})$ are expected to exhibit a normal behaviour in both uniform and non uniform interacting Bose gases.

[1] M.H. Anderson, J.R. Ensher, M.R. Matthews, C.E. Wieman and E.A. Cornell, Science 269, 198 (1995); K.B. Davis, M.-O. Mewes, M.R. Andrews, N.J. van Druten, D.S. Durfee, D.M. Kurn and W. Ketterle, Phys. Rev. Lett. 75, 3969 (1995); C.C. Bradley, C.A. Sackett and R.G. Hulet, Phys. Rev. Lett. 78, 985 (1997).
[2] BEC on line bibliography, Georgia Southern University, www-address http://amo.phy.gasou.edu:80/bec.html

[3] R.M. Ziff, G.E. Uhlenbeck and M. Kac, Phys. Rep. 32, 169 (1977).

[4] E.H. Hauge, Physica Norvegica 4, 19 (1969).

[5] I. Fujiwara, D. ter Haar and H. Wergeland, J. Stat. Phys. 2, 329 (1970).

[6] H.D. Politzer, Phys. Rev. A 54, 5048 (1996).

[7] M. Gajda and K. Rzążewski, Phys. Rev. Lett. 78, 2686 (1997); P. Navez, D. Bitouk, M. Gajda, Z. Idziaszek and K. Rzążewski, Phys. Rev. Lett. 79, 1789 (1997).

[8] S. Grossmann and M. Holthaus, Report No. condmat/9709045 and Report No. cond-mat/9709065.

[9] N. Bogoliubov, J. Phys. USSR 11, 23 (1947).

[10] R.A. Ferrel, N. Meynhard, H. Schmidt, F. Schwabl and P. Szépfalusy, Ann. Phys. (N.Y.) 47, 565 (1968).

[11] The distinction between canonical and grand canonical ensemble for the calculation of $\left\langle\delta N_{0}^{2}\right\rangle$ is crucial only in the ideal Bose gas. The presence of interactions stabilizes the fluctuations of $N$ and ensures the equivalence, in the thermodynamic limit, of the two ensembles also for the low- $T$ behaviour of $\left\langle\delta N_{0}^{2}\right\rangle$.

[12] In the case of the ideal Bose gas one can easily work also with different boundary conditions [3].

[13] J. Gavoret and P. Nozières, Ann. Phys. (N.Y.) 28, 349 (1964); P.C. Hohenberg and P.C. Martin, Ann. Phys. (N.Y.) 34, 291 (1965); A. Griffin, Excitations in a BoseCondensed Liquid (Cambridge University Press, New York, 1993).

[14] S. Giorgini, L. P. Pitaevskii and S. Stringari, Phys. Rev. B 46, 6374 (1992).

[15] E.M. Lifshitz and L.P. Pitaevskii, Statistical Physics, Part 2 (Pergamon, Oxford, 1981), eq. (87.6).

[16] For an ideal gas in the grand canonical ensemble the same method gives $\left\langle\delta N^{2}\right\rangle(T=0)=\langle N\rangle$, as can be directly obtained from eq. (11) with $\nu(\mathbf{r})=\delta(\mathbf{r})$.

[17] S.R. de Groot, G.J. Hooyman and C.A. Ten Seldam, Proc. R. Soc. (London). A 203, 266 (1950); V. Bagnato, D.E. Pritchard and D. Kleppner, Phys. Rev. A 35, 4354 (1987).

[18] S. Giorgini, L.P. Pitaevskii and S. Stringari, Phys. Rev. Lett. 78, 3987 (1997), and Report No. condmat/9704014, to appear in J. Low Temp. Phys..

[19] K. Damle, T. Senthil, S.N. Majumdar and S. Sachdev, Europhys. Lett. 36, 7 (1996).

[20] S. Stringari, Phys. Rev. Lett. 77, 2360 (1996).

[21] D.S. Jin, J.R. Ensher, M.R. Matthews, C.E. Wieman and E.A. Cornell, Phys. Rev. Lett. 77, 420 (1996); M.O. Mewes, M.R. Andrews, N.J. van Druten, D.M. Kurn, D.S. Durfee, C.G. Townsend and W. Ketterle, Phys. Rev. Lett. 77, 988 (1996).

[22] W. C. Wu and A. Griffin, Phys. Rev. A 54, 4204 (1996). 